\title{
Chemical Classification of Discrete Second Phases in the SEM
}

John J. Friel*

*Princeton Gamma-Tech, 1026 Rt. 518, Rocky Hill, NJ 08553

Image analysis in the SEM is generally slower than in the LOM, but the SEM affords more contrast mechanisms. In some cases, composition can be inferred from atomic number contrast in the backscattered electron (BSE) signal, but the x-ray signal is generally necessary for unambiguous chemical classification. By combining $x$ rays with one of the electron imaging signals, image analysis and microanalysis can be performed simultaneously. Automatic chemical classification is a term that is generally applied to the process of finding features in an image and driving the electron beam back to them for chemical analysis and classification according to a predetermined set of rules. The first systems were designed for particles when digital beam control first became available [1].

Image analysis is used both to measure size and shape and to filter the features before collecting compositional data. For example, one could define types A, B, and C inclusions as features with an aspect ratio $>\mathrm{n}$, and define Type $\mathrm{D}$ globular oxides by a range of circularity. All other features would be excluded, before driving the electron beam to the features for chemical analysis. Fig. 1 shows a duplex nonmetallic inclusion with both sulfides and silicates in it. Chemical classification data taken from an AISI S7 sample in the SEM, and consisting of 30 fields containing inclusions is shown in Table 1. Classification according to ASTM E 45 is done in the LOM, but ASTM E 2142 requires SEM data for the direct determination of composition [2]. Of particular interest is the ability to classify duplex inclusions chemically. Sulfide/silicate duplex inclusions are reported as duplex $\mathrm{S} / \mathrm{Si}$ in Table 1. Other discrete second phases could be similarly analyzed and classified based on the alloy system and the purpose of the analysis.

An example taken from extractive metallurgy is shown in Fig. 2. The bright regions in the SE image are sulfides, but the silver-bearing cubic tetrahedrite, $(\mathrm{Cu}, \mathrm{Ag})_{10}(\mathrm{Fe}, \mathrm{Zn})_{2}(\mathrm{Sb}, \mathrm{As})_{4} \mathrm{~S}_{13}$, is indistinguishable from the tetragonal chalcopyrite, $\mathrm{CuFeS}_{2}$, that contains no silver. The pseudocolored image on the right in Fig. 2 shows the sulfides as green, but $\mathrm{x}$ rays are required to distinguish the economic mineral from the other sulfides. Rules for classifying the minerals are defined empirically, but the dataset can be reclassified after collection to optimize the classification. Rules can be added, deleted, or modified, and they can be based on: gross or net counts, interelement ratios, peak/background ratios, or a combination thereof. The results for the sulfide ore are given in Table 2. The data show that the Ag-bearing sulfide grains outnumber those without Ag by about 2:1. Fig. 3 shows an EDS spectrum for tetrahedrite extracted from a spectrum image after automatically sorting into phases by the method of recursive pixel allocation [3]. The method of chemical classification in the SEM works for any discrete second phase that can be isolated in the microstructure and uniquely classified chemically.

\section{References}

[1] S. Ekelund and T. Werlefors, SEM III (1976) 417.

[2] E 2142 Annual book of ASTM standards vol. 03.01, ASTM West Conshohocken.

[3] J. J. Friel and R. Batcheler, Microsc. Microanal. 8 (Suppl 2) (2002) 350. 


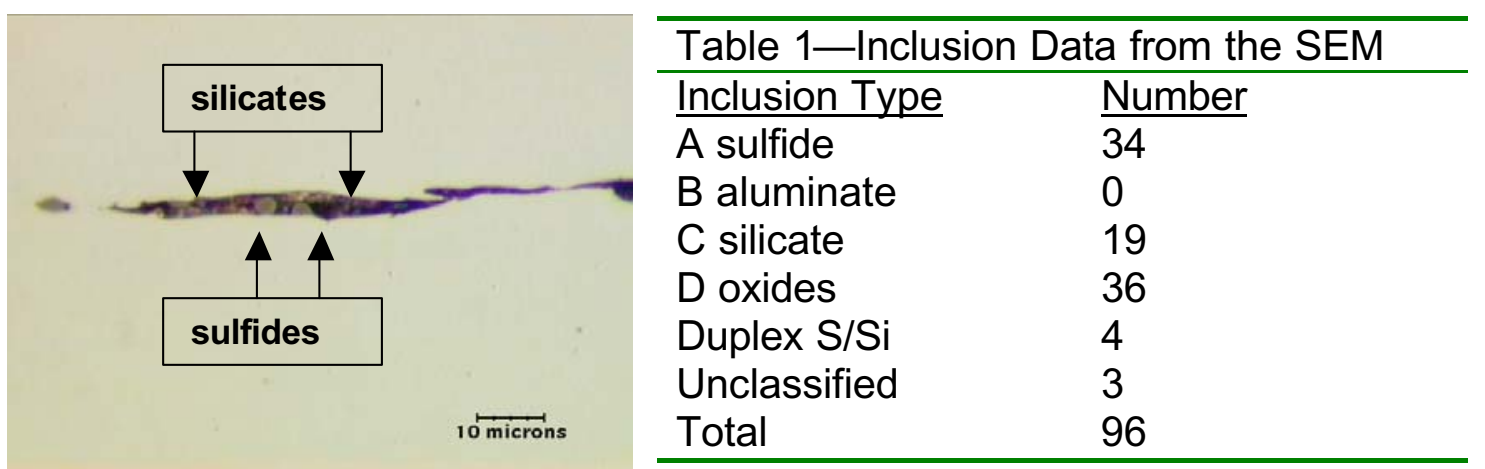

Fig 1. LOM image of a duplex inclusion in steel.

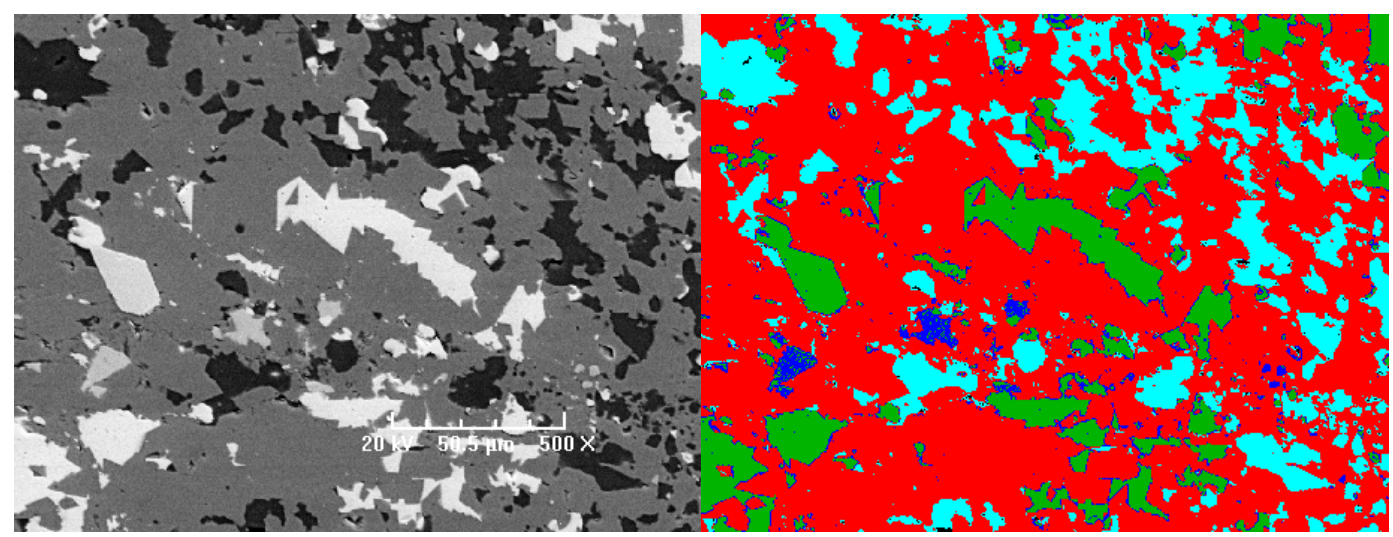

Fig 2. SE image of sulfide $\mathrm{Cu} / \mathrm{Ag}$ ore with grayscale (left) and pseudocolor (right). The cyan phase is quartz; the red phase is mostly siderite, and the green includes both tetrahedrite and chalcopyrite. Blue is arsenopyrite.

\begin{tabular}{|c|c|c|}
\hline Mineral & Classification Rules & No. of grains \\
\hline$\overline{\text { Tetrahedrite (Ag-rich) }}$ & Ag p/b>0.2 AND S $p / b>0.4$ AND Sb $p / b>1.0$ & 23 \\
\hline Chalcopyrite (Ag-poor) & Cu $p / b>1.0$ AND Fe $p / b>0$ & 12 \\
\hline Quartz & Si:Fe $>3$ & 11 \\
\hline Siderite & Fe $p / b>1.0$ AND C $p / b>1.0$ AND Cu $p / b<1.0$ & 39 \\
\hline Clinopyroxene & Fe $p / b>1.0$ AND Si $p / b>1.0$ AND Ca $p / b>0.5$ & 4 \\
\hline Unclassified & Other & 2 \\
\hline Total & & 91 \\
\hline
\end{tabular}

- Tetrahedrite.pgt

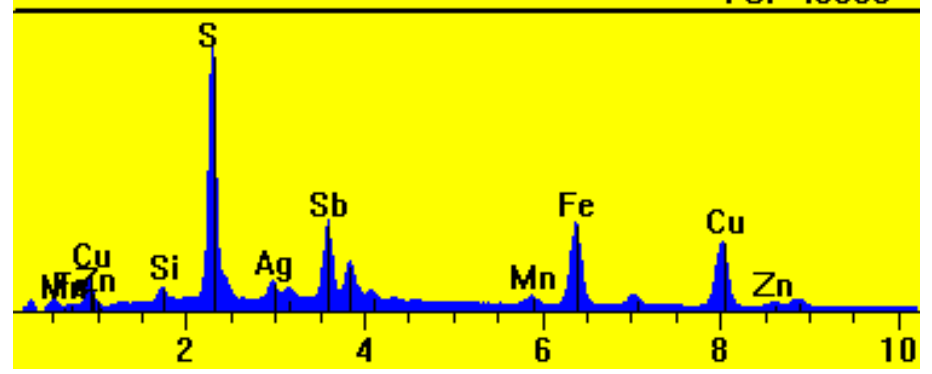

Fig 3. Sum spectrum of all Ag-bearing tetrahedrite grains. 